\title{
A Tunable and Highly-Efficient Plasmonic Band-Stop Filter at Telecom Wavelengths
}

\author{
Seyed Morteza Ebadi *, Student Member, IEEE, and Jonas Örtegren \\ Department of Natural Sciences, Mid Sweden University, SE-85170 Sundsvall, Sweden \\ E-mail: *seyed-morteza.ebadi@miun.se
}

\begin{abstract}
This paper reports design and numerical results of an ultra-compact, efficient and tunable plasmonic bandstop filter based on a metal-insulator-metal waveguide at optical channels. Furthermore, a wide-bandgap can be easily achieved by adjusting the width of the of the stub resonator.

\section{Introduction}

Surface plasmon polaritons (SPPs), which is the coupling of the oscillation of free electrons to electromagnetic surface waves at a metal and dielectric interface, may overcome the diffraction limit of light and provide guidance and confinement of light at deep subwavelength scales [1]. This coupling leads to a number of unique characteristics that may be hard to find elsewhere, such as greatly contributing to the size reduction of optical circuits to nano-meter scales, and may be regarded as the technology that can incorporate both photonics and electronics circuits on a single chip. SPP technology may thereby feature high-efficiency, compact, yet cost-effective solutions for next generation of photonic integrated circuits (PICs) [2,3]. Generally, there are two kinds of plasmonic based waveguides: insulatormetal-insulator (IMI) and metal-insulator-metal (MIM). The MIM structure may be considered as promising for incorporation into PICs, since it maintains an acceptable trade-off between mode confinement and loss. Hence, a number of plasmonic-based structures such as sensors [4], splitters [5], modulators [6], and so forth have been suggested. One of the main and important components in PICs is optical filters that are designed to pass light at a desired frequency range, while blocking other frequencies. In fact, there are several applications of optical filters such as fluorescence microscopy, imaging systems, wavelength-division multiplexing (WDM) in optical communication systems, LIDAR (Light Detection and Ranging) systems, and dispersion compensation that can be mentioned to demonstrate their extensive functionalities in various circuits. It should be pointed out that the focus of our study is to form a band-stop filter spectrum at optical channels, and thus, all parameters are optimized for best possible performance, considering the resonance wavelength position, size, and the efficiency of the structure. Consequently, to promote the advancement and miniaturization of optical circuits, we propose a tunable and extremely-compact band-stop filter that functions at the technologically important telecom wavelengths. The simulation results confirm that wide-bandgap can be readily attained through modulation of structural parameters.
\end{abstract}

\section{Device of the Prpoposed Tunable Band-Stop Plasmonic Filtert and Simulation Results}

(a)

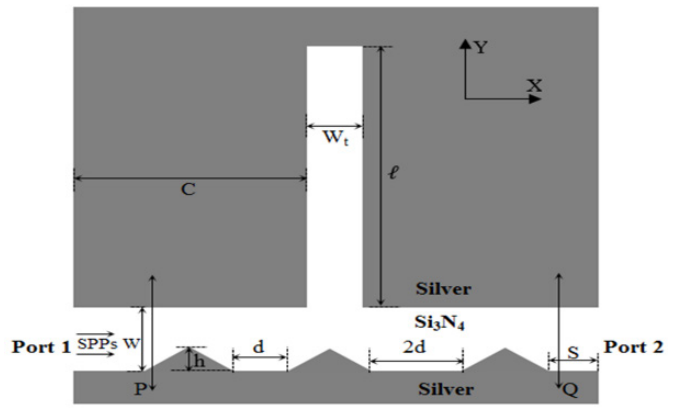

(b)

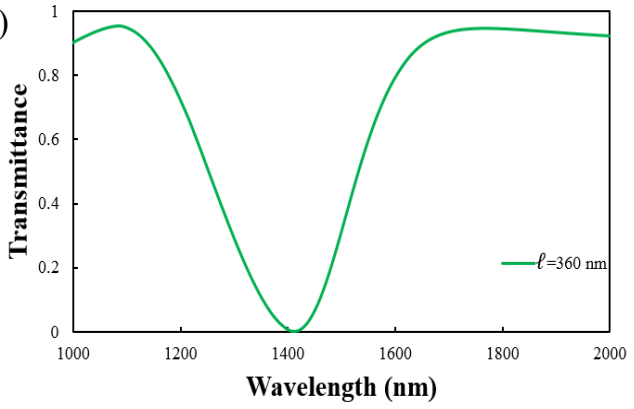

Fig. 1. (a) Schematic configuration of proposed band-stop plasmonic filter. (b) Transmission profile of the band-stop wavelength filter for $\mathrm{W}=55 \mathrm{~nm}, \mathrm{C}=200 \mathrm{~nm}, \mathrm{~S}=50 \mathrm{~nm}, \mathrm{~d}=60, \mathrm{~h}=15 \mathrm{~nm}$, and $\ell=360 \mathrm{~nm}$.

Figure 1(a) schematically exhibits the configuration of the proposed nanoplasmonic band-stop wavelength filter. The structure is made of two layers of silver, whose complex permittivity has been taken from tabulated data of Johnson and Christy [7], whereas the insulator layer is assumed to be Silicon nitride with refractive index of 1.99 [8]. The width of the waveguide $\mathrm{W}$ is set to be substantially smaller than the wavelength of the incident light, in order to assure that only the fundamental TM mode can exist in the structure. The TM mode is excited by a dipole source. The grid sizes are set to be $5 \mathrm{~nm} \times 5 \mathrm{~nm}$ along the $\mathrm{x}$ and $\mathrm{y}$ directions, respectively. Two power monitors $\mathrm{P}$ and $\mathrm{Q}$ are set, respectively, at equal distance from the center of the waveguide to detect the incident and transmitted power in the device. The transmission 
is then described as T=Pout/Pi [9]. We have utilized a full-wave EM tool, CST MWS, with frequency domain solver, which is based on the finite element method (FEM), to attain the numerical results in this letter. Figure 1 (b) illustrates the simulation results of the ultracompact and tunable band-stop filter for the physical parameters $\mathrm{W}=55 \mathrm{~nm}$, (the width of the waveguide), $\mathrm{C}=200 \mathrm{~nm}$ (the distance between the bus waveguide and the stub), $\mathrm{S}=50 \mathrm{~nm}$ (the distance between the port and the triangular cavities), $\mathrm{d}=60 \mathrm{~nm}$ (the distance between the triangular cavities), $\ell=360 \mathrm{~nm}$ (the length of the stub), $\mathrm{W}_{\mathrm{t}}=50 \mathrm{~nm}$ (the width of stub), and $\mathrm{h}=15 \mathrm{~nm}$ (the height of the triangular cavities). It is obvious that the transmission is nearly zero at a wavelength of $1400 \mathrm{~nm}$ for the determined stub length, which indicates that the resonance wavelength is formed through coupling between the stub resonator, triangular cavities and the bus waveguide.

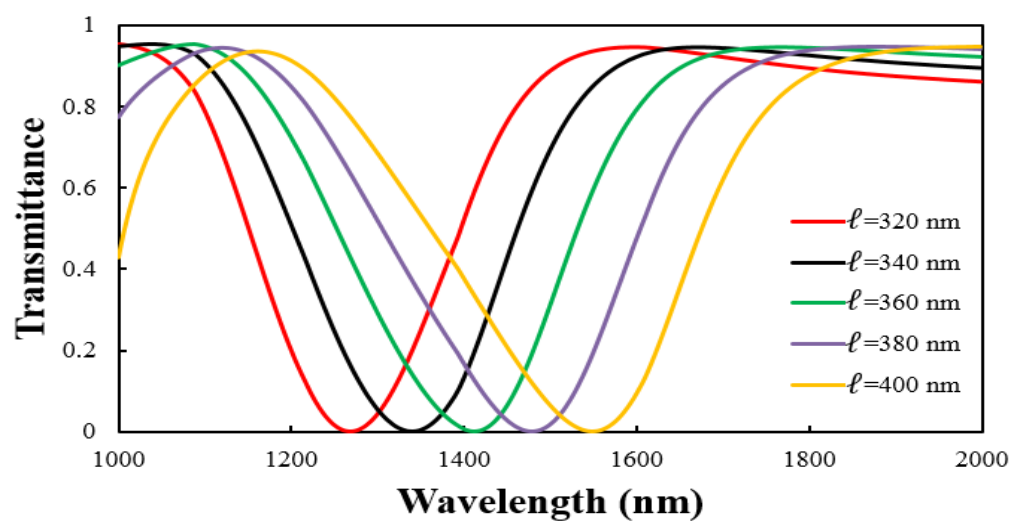

Fig. 2. The transmission spectra as a function of the wavelength for the suggested band-stop wavelength filter with various lengths of the stub resonator.

Figure 2 illustrates the numerical results of the transmission profile of the proposed tunable band-stop wavelength filter at the telecom wavelengths for $\ell=320 \mathrm{~nm}, 340 \mathrm{~nm}, 360 \mathrm{~nm}, 380 \mathrm{~nm}$, and $400 \mathrm{~nm}$, whereas all other parameters of the structure are the same as in Fig. 1 (a). It is demonstrated that by reducing the length of the stub, the bandgap moves to shorter wavelengths, and vice versa. For instance, it was revealed that while $\ell=400 \mathrm{~nm}$, the corresponding transmission of the cut-off wavelength that is $1549.8 \mathrm{~nm}$, is 0.001 . We have thereby shown that through modifying the width of the stub resonator, $\ell$, one can selectively modulate the bandgap wavelength of the filter.

\section{Conclusion}

In summary, the proposed band-stop filter is more compact and efficient compared to similar studies [10- 11], and may significantly contribute to miniaturization of optical circuits, and find applications in next generation of PICs as well as in on-chip optical circuits.

\section{References}

[1] Brongersma, Mark L., and Vladimir M. Shalaev. "The case for plasmonics." science 328.5977 (2010): $440-441$.

[2] Ozbay, Ekmel. "Plasmonics: merging photonics and electronics at nanoscale dimensions." science 311.5758 (2006): $189-193$.

[3] Maier, Stefan Alexander. Plasmonics: fundamentals and applications. Springer Science \& Business Media, 2007.

[4] M. S. Islam et al., "Dual-polarized highly sensitive plasmonic sensor in the visible to near-IR spectrum," Optics express, vol. 26, no. 23, pp. 30347-30361, 2018.

[5] Ye, Yichen, et al. "Design of a Novel Plasmonic Splitter With Variable Transmissions and Selectable Channels." IEEE Transactions on Nanotechnology 18 (2019): 617-625.

[6] Heni, Wolfgang, et al. "High speed plasmonic modulator array enabling dense optical interconnect solutions." Optics express 23.23 (2015): 29746-29757.

[7] P. B. Johnson and R.-W. Christy, "Optical constants of the noble metals," Physical review B, vol. 6, no. 12, p. 4370, 1972.

[8] https://refractiveindex.info/?shelf=main\&book=Si3N4\&page=Luke. (Accessed 16 February 2021).

[9] S. M. Ebadi, et al, "High-Efficiency Nanoplasmonic Wavelength Filters Based on MIM Waveguides," IEEE Photonics Technology Letters, vol. 28 , no. 22 , pp. 2605-2608, 15 Nov.15, 2016.

[10] Zhang, Zhaojian, et al. "Plasmonic filter and demultiplexer based on square ring resonator." Applied Sciences 8.3 (2018): 462.

[11] S. M. Ebadi, et al, "A Multipurpose and Highly-Compact Plasmonic Filter Based on Metal-Insulator-Metal Waveguides," IEEE Photonics Journal, vol. 12, no. 3, pp. 1-9, June 2020. 